addition of a definite quantity of a carbohydrate, which at the same time increases its nutritive value and renders it a perfect food for infants and invalids. It is rich in albuminoids, but contains no excess of salts or nitrogenous waste matter. Consequently, it usually agrees and may be taken with safety by those whose digestive powers are weak. It is not a dear preparation.

One teaspoonful added to a "lemon-squash," or to one tablespoonful (more or less) of lime-juice or lemon-juice cordial, with cold water or soda water, makes a pleasant and nourıshing summer drink. Milk may be substituted for lime-juice cordial, if so desired, and this will be found an equally palatable and refreshing beverage.

\title{
Toilet Lanolin.
}

This preparation has been introduced by the enterprising firm of Burroughs, Wellcome, \& Co. It is elegantly perfumed, and is sold in compressible capsules, which are most convenient and cleanly in use.

\section{Coca Preparations.}

Messrs. Armbrecht, Nelson, \& Co., 2 Duke-street, Grosvenor-square, London, have placed in our hands specimens of several of their preparations of coca leaves (Erythroxylon coca). First of all are samples of coca wine. This is made of five sorts-sweet Malaga, in blue cases, suitable for ladies and children; Burgundy, in red cases, for the gouty and dyspeptic; so-called "medium," in drab-coloured cases, said to be the pleasantest of all and to contain most coca; lastly, coca sherry and coca port. Besides these, there is a very palatable effervescing wine, called "Vin Mousseux de Coca," or "Coca Champagne." This costs 56s. a dozen in quarts, or 33s. a dozen in pints. It seems to be a sound wine, and is very pleasant to the palate and exhilarating.

The other preparations are the coca extract and lozenges. The former is described as a powerful nerve stimulant. In small doses (five to fifteen drops before each meal) it increases appetite and promotes digestion. In similar doses, given at bedtime, and repeated once after an hour if so required, it produces sound and refreshing sleep in cases of sleeplessness from exhaustion and fatigue. The lozenges, which are not very palatable, are recommended instead of the wine where it is not convenient to take the latter. They contain the extract of the green coca leaves, and are indicated in cases of loss of voice.

\section{ERRATUM.}

In the number of the Journal for June, 1890 (Vol. LXXXIX., No. 222, Third Series), page 510, line 10 from the top, for "Schlafkrankenheit," read "Schlaftrunkenheit." 